Revue européenne des migrations internationales

vol. $19-n^{\circ} 3 \mid 2003$

Moyen-Orient : mutations récentes d'un carrefour migratoire

\title{
Le « retour du nombre » : permanences et limites de la stratégie territoriale israélienne
}

\section{William Berthomière}

\section{(2) OpenEdition \\ Journals}

Édition électronique

URL : https://journals.openedition.org/remi/475

DOI : $10.4000 /$ remi.475

ISSN : $1777-5418$

Éditeur

Université de Poitiers

Édition imprimée

Date de publication : 12 décembre 2003

Pagination : 73-93

ISBN : 2-911627-35-0

ISSN : 0765-0752

Référence électronique

William Berthomière, «Le « retour du nombre » : permanences et limites de la stratégie territoriale israélienne », Revue européenne des migrations internationales [En ligne], vol. 19 - n³ | 2003, mis en ligne le 09 juin 2006, consulté le 14 avril 2022. URL : http://journals.openedition.org/remi/475 ; DOI : https://doi.org/10.4000/remi.475

Ce document a été généré automatiquement le 14 avril 2022

(c) Université de Poitiers 


\title{
Le « retour du nombre»: permanences et limites de la stratégie territoriale israélienne
}

\author{
William Berthomière
}

1 Le nombre. Au cours de la décennie quatre-vingt-dix, il semblait avoir perdu sa place ou plus justement s'être figé en un seul : 1993, année des «accords d'Oslo». Mais, depuis le déclenchement de la seconde Intifada, il reprend sa place avec toute sa gravité : 3177 le nombre de personnes ayant perdu la vie depuis septembre 2000 (2 625 Palestiniens et 865 Israéliens $^{1}$ ), appel à l'entrée d'un nouveau million d'immigrants par Ariel Sharon ${ }^{2}$ ou encore multiplication des projections démographiques mettant en concurrence les populations juive et palestinienne en 2005, 2010, 2020, 2050... Le retour $\mathrm{du}$ nombre souligne ainsi tristement la faiblesse des diplomaties occidentales et orientales, qui n'ont su accompagner et soutenir un processus de paix fragilisé dès ses premières heures par les pièges internes qu'il recelait (Laurens, 1999 ; Legrain, 2000 ; Debié et al., 2001).

2 Pour Israël, cette tentation du nombre a pris corps dans l'assurance apportée par l'entrée de près d'un million d'immigrants sur la décennie 90 , notamment venus d'exURSS. Ce dynamisme migratoire a pu ainsi être doublement interprété. Premièrement, il a affirmé la valeur cardinale du sionisme en replaçant - d'une certaine manière Israël dans une temporalité $d u$ « retour » ou le poète Nathan Alterman écrivait :

«C'est si bon d'être un million

A tous les voir, tes yeux se mouillent

De larmes brillantes. Pourquoi cela?

Car nous te l'avions dit, mon frère - les statistiques

Ne sont pas toujours arides $"^{3}$

Deuxièmement, cet afflux de migrants a pu alimenter une stratégie territoriale recherchant le fait accompli et où résonnent aujourd'hui avec toute leur force les propos d'Ytshak Shamir lorsqu'il déclarait dès 1990 qu'une "grande immigration nécessite un grand Israël $»^{4}$. Néanmoins, la situation actuelle n'est en rien comparable à celle qui prévalait au lendemain de la première Intifada et de la recrudescence de 
l'immigration en Israël. Les sociétés palestinienne et israélienne ont connu de véritables changements de leurs structures sociodémographiques. La première, par la territorialisation de son autorité politique en Palestine, fait face- non sans difficultés - à une reconfiguration des rapports entre Palestiniens de l'intérieur et de l'extérieur (Berthomière et al., 2002). La seconde voit son projet de construction nationale subir d'importantes perturbations sociales que résume - en partie - le débat entre post et néo-sionistes.

Entre « societe militaire et société civile » : un processus de construction nationale perturbé

4 Pour l'ensemble de la communauté internationale, la décennie 90 constitue un véritable tournant dans l'histoire du conflit israélo-palestinien. Pour les Israéliens, la poignée de main entre Yasser Arafat et Ytshak Rabin est certes un moment fort de leur histoire mais cette décennie ne saurait se résumer à cet événement. Israël a connu en son sein de profondes reconfigurations sociales qui ont perturbé son processus de construction nationale. Au premier chef, la succession des vagues migratoires et surtout leur diversité ont fortement marqué cette société en formation. Le profil migratoire d'Israël au fil de la cinquantaine d'années d'immigration permet de mieux comprendre l'ampleur et la teneur des changements internes à la société israélienne depuis 1948 (figure 1).

Figure 1 : Immigration en Israël par continent d'origine (1948-2002)[afficher]

Impulsé par le sionisme politique, Israël s'est construit sur l'immigration des « exilés » dès la fin $d u x^{e} x^{e}$ siècle et, de façon plus conséquente, avec les grandes vagues migratoires consécutives à la création de l'État en 1948. Les Pères fondateurs de l'État ont érigé Israël en se donnant pour objectif la « fusion des exilés » (mizzoug galouyoth) d'où devait naître un peuple juif ressourcé par le retour à la terre et l'établissement sur les lieux de sa patrie ancestrale. En à peine plus d'une cinquante d'années d'existence, l'Etat d'Israël a vu immigrer près de trois millions de Juifs. Des milliers de Juifs rescapés de la Shoah et originaires du Moyen-Orient ont constitué les premiers contingents de la vague massive d'immigrations qu'a connu le pays au cours de ces quinze premières années. A ces migrants originaires d'Europe et du Moyen-Orient ont succédé ceux venus des rives maghrébines de la Méditerranée. Les Juifs marocains, qui ont composé une large part de ce nouvel effectif migrant, ont clos la temporalité du retour dans laquelle Israël était inscrit depuis 1948 (figure 2). 
Figure 2 : Schématisation des contextes migratoires et sociaux d'Israël (1948-2003)

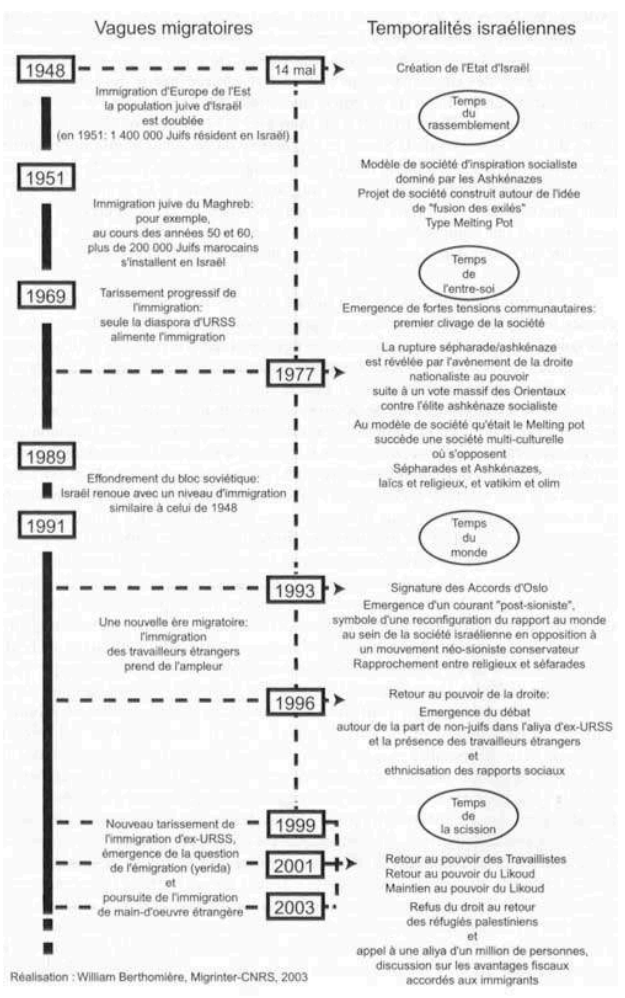

6 La multiplication des conflits armés avec les pays voisins et la nécessité de sécuriser les espaces périphériques du pays ont poussé les Pères fondateurs de l'État sur la voie d'une politique d'aménagement territorial qui a reposé sur la dispersion des nouveaux arrivants afin de mieux contrôler le territoire. Fondée sur l'idée, exprimée par Golda Meir, que "la frontière de l'Etat se trouve là où sont les Juifs", la création d'un ensemble d'établissements de populations au sein de l'espace rural israélien s'est faite en laissant de coté la dimension sociale que requerrait alors l'installation de populations déracinées. La création, dans l'urgence, d'un tissu humain dans les espaces périphériques d'Israël (le Néguev au sud, et la Galilée au nord) s'est trouvée génératrice d'une fracture socio-spatiale. Très rapidement, les difficultés rencontrées par les aménageurs d'État pour assurer l'essor économique des villes de développement ont eu pour effet de dessiner une ligne de fracture entre ces régions périphériques et le cœur du pays constitué par la région métropolitaine de Tel-Aviv. Peuplées dans leur très grande majorité par des Juifs orientaux (venus d'Afrique du Nord ou du Moyen-Orient), les populations des territoires périurbains et ruraux ont progressivement inscrit leur représentation des inégalités sociales dans une grille de lecture articulée autour de la ligne de fracture "séfarade-ashkénaze». L'affaiblissement migratoire de la fin des années soixante, qui aurait pu permettre, par ce repli sur lui-même de l'Etat, la réalisation de la «fusion des exilés", a souligné un pays qui s'est trouvé progressivement confronté à l'émergence d'un "problème ethnique " (figure 2; Eisenstadt, 1985, 1986 ; Poirier, 1998). L'année 1977 marque alors doublement cette temporalité puisqu'elle est l'occasion, lors des élections de mai, d'un vote de rejet exprimé par les séfarades envers l'establishment ashkénaze et, avec l'accession au pouvoir de la droite nationaliste qui en découle, le moment d'une intensification de la politique de colonisation des territoires occupés depuis 1967 (notamment sous l'impulsion du ministre de l'Agriculture - puis de la Défense - d'alors, Ariel Sharon). 
Avec cette arrivée au pouvoir du Likoud se mettent en place les premiers éléments de complexification des clivages sociaux d'Israël où s'entrecroisent les facteurs socioéconomiques, politiques, communautaires et religieux (Ram, 1995; Klein, 1999). Les vagues migratoires des années quatre-vingt et surtout quatre-vingt dix avec l'arrivée de Juifs éthiopiens et de près d'un million de Juifs d'ex-URSS (figure 1) se sont inscrites dans ce modèle de société et ont participé à l'affirmation de ce processus de complexification des rapports sociaux, notamment par l'importance croissante des partis dits ethniques comme le Shas (parti séfarade orthodoxe) ou Israel Be'Aliya (parti russe créé par Nathan Charansky).

7 La décennie quatre-vingt dix s'ouvre donc dans un contexte social totalement différent de celui des lendemains de la guerre de 1967. Le nationalo-mysticisme, développé notamment par le Gouch Emounin ${ }^{5}$, constitue aujourd'hui le pôle irrédentiste d'Israël en opposition totale avec celui qualifié de post-sioniste (Silberstein, 1999; Dieckhoff, 2001). Israël se définit comme une société en tension, fruit d'une israélite fortement marquée par une instrumentalisation de l'ethnicité et - selon Baruch Kimmerling par un «militarisme culturel ", notablement consolidé par l'évènement qu'a constitué la première Intifada (1987) (Ben-Eliezer, 1999 ; Kimmerling, 2001).

L'identité nationale israélienne, qui va « accueillir » le processus de paix, se caractérise par un état de crise où le pays se trouve, d'un côté, propulsé dans une temporalité rythmée par le phénomène de globalisation, de l'autre, réinscrit dans la temporalité du rassemblement avec l'entrée de milliers de juifs ex-soviétiques et éthiopiens. L'émergence d'un temps du monde (figure 2), qui pour les néo-fondamentalistes symbolise "un Israël matérialiste et occidentalisé qui aurait trahi sa mission nationale " (Ram, 2000), prend ainsi force en Israël sous différentes formes : le débat suscité par les Nouveaux historiens, celui sur le post-sionisme (Greilsammer, 1998 ; Dieckhoff, 2001)

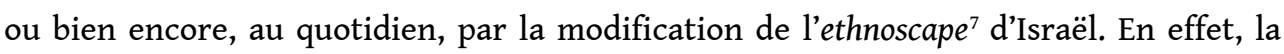
spirale de violence qui s'élève au milieu des années quatre-vingt dix, suite à l'enlisement $\mathrm{du}$ processus d'Oslo, a eu pour conséquences d'affaiblir le capital économique palestinien par les fermetures répétées et de plus en plus prolongées des frontières d'Israël aux travailleurs palestiniens ${ }^{8}$ mais aussi d'obliger Israël à trouver à l'extérieur une main d'oeuvre de remplacement. Avec les appels à main-d'œuvre étrangère et de part son fort potentiel économique en Méditerranée orientale, Israël s'est donc trouvé inscrit dans le système migratoire international et a vu progressivement sa main-d'oeuvre palestinienne être remplacée par des travailleurs émigrés - réguliers et irréguliers - venus de dizaines de pays (Berthomière, 1999) (tableau 1). 
Tableau 1 : Travailleurs étrangers et palestiniens des Territoires occupés et du sud-Liban (moyenne mensuelle) selon le secteur d'activité (1994-2002)

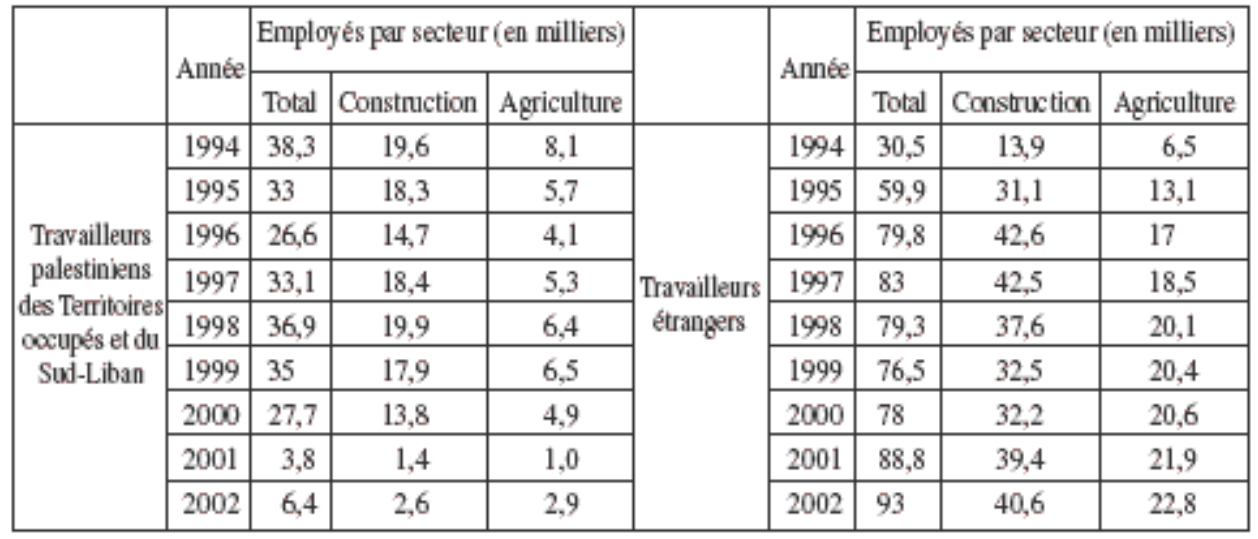

Source : Statistical Abstract of Israel, 2003

9 Comme les vives tensions suscitées par la multiplication des travaux réalisés par les «nouveaux historiens » comme Ilan Pappé, l'entrée de travailleurs étrangers (et leur possible installation) a été l'occasion de vives tensions au sein de la société israélienne. Des articles intitulés «Ne tombez pas amoureux d'un travailleur étranger » ou «Le Pen parle hébreu » ou plus récemment la mise en place d'une police de l'immigration et l'accroissement des expulsions ${ }^{9}$ ont souligné les tensions internes que connaît le pays alors qu'il est engagé dans un processus de négociations qui doit mener à une paix durable (Berthomière, 2003b; Ellman et al., 2003). Replacés dans le temps long, ces éléments soulignent la complexité du processus de construction nationale d'Israël et éclaire d'autant mieux les conséquences internes de la présence israélienne au-delà de la Ligne verte. Israël apparaît comme «tiraillé » entre ces deux pôles - post et néosionistes - et voit toute tentative de négociation sociale neutralisée par son incapacité à faire évoluer un processus de construction nationale "empêtré " dans un pseudo statu quo entre société civile, religieuse et militaire, en fait très en faveur de cette dernière.

D'une manière presque caricaturale, au lendemain de la première Intifada alors même que la société militaire est poussée à la négociation par la libéralisation économique du pays mais surtout par sa perte de dynamisme démographique (Berthomière, 2000), le sursaut migratoire créé par l'effondrement de l'Union soviétique fait table rase de cette possible ouverture. Pour reprendre une réflexion de Nicole Lapierre ${ }^{10}$, Israël se trouve alors réinscrit dans le « mythe du retour, du temps cyclique qui s'ancre dans l'évidence immuable d'un donné originel et abolit l'histoire » et voit son Premier ministre d'alors, Ytshak Shamir, réaffirmer l'idée que la « la frontière se trouve là où habitent des juifs, et non pas sur un tracé de carte » (Kapeliouk, 1975) ${ }^{11}$.

La tentation du nombre ou l'inévitable impasse stratégique?

Dans ce contexte socio-politique perturbé du début des années quatre-vingt dix, le retour à un fort niveau d'immigration a servi les intérêts de la «société militaire » et a clairement replacé Israël dans la problématique « immigration-territoire » comme l'ont souligné les propos d'Ytshak Shamir (cf. supra). La politique démographique d'Israël s'est de nouveau engagée dans une logique de stratégie territoriale telle qu'avait pu la définir David Ben Gourion lorsqu'il déclarait : «Nous avons conquis des territoires, mais que ce soit dans le Néguev, en Galilée ou à Jérusalem, leur importance reste mineure tant qu'ils ne sont pas peuplés de colonies. La colonisation, voilà la véritable conquête. [...] L'avenir de l'État 
dépend de l'immigration" (Segev, 1998). Largement dotés en plan de peuplement, de maîtrise démographique ou de conquête territoriale (Dieckhoff, 1987; Legrain, 1996), ces espaces «stratégiques" ont donc bénéficié d'une attention particulière, la vague des nouveaux immigrants ex-soviétiques pouvant y pallier ou accroître la croissance naturelle de la population juive. Cette politique a reposé principalement sur l'intensification de la politique de constructions et l'aide à l'accession à la propriété chez les nouveaux immigrants (Portnov, 1998). Les dirigeants israéliens ont donc chercher à installer ceux-ci dans les régions périphériques notamment en Galilée ou le plan de «judaïsation» de la région était en échec (Falah, 1991 ; Yiftachel, 1992). La Cisjordanie a été également concernée par ce projet puisque les plus importantes de ses colonies (principalement les cités-dortoirs de Jérusalem et de Tel-Aviv) ont vu passer leur part de constructions en chantier de $3 \%$ en 1990 à $6 \%$ en 1991 et $19 \%$ en 1992 (Alterman, 2002). Malgré la diversité des incitations économiques à la construction dans ces espaces et le large éventail des aides accordées aux nouveaux immigrants (ce qui n'a pas été sans créer de tensions avec les populations défavorisées d'Israël), le binôme « immigration-stratégie » a montré des signes de faiblesse.

Un binôme « immigration-territoire » qui s'épuise

À l'échelle des frontières d'avant 1967, il ressort qu'Israël n'a pu redynamiser sur le long terme la croissance démographique de la Galilée ${ }^{12}$. L'immigration, notamment d'ex-URSS ${ }^{13}$, n'a pas été "l'arme démographique » qui aurait permis la "judaïsation complète" de cette région. Dans son article "Israeli "Judaization" Policy in Galilee» (1991), Walid-Ghazi Falah concluait que l'immigration d'ex-URSS risquait d'altérer de manière notable la réalité démographique de cette région, une décennie plus tard nous pouvons constater que cette immigration a certes permis un essor considérable de la présence juive en Galilée (tableau 2) mais qu'il a été nettement contrebalancé par une forte croissance naturelle des populations palestiniennes (environ 3,4 \% par an (Yaffe et al., 2002) ${ }^{14}$.

Tableau 2 : Populations arabe et juive dans le district Nord (1948-2002)

\begin{tabular}{|c|c|c|c|}
\hline Année & Population juive & Population arabe & Ecart de population \\
\hline 1948 & 53400 & 90600 & -37200 \\
\hline 1972 & 255700 & 217600 & 38100 \\
\hline 1983 & 327000 & 329000 & -2000 \\
\hline 1989 & 364800 & 397900 & -33100 \\
\hline 1994 & 458700 & 468100 & -9400 \\
\hline 1995 & 471900 & 475000 & -3100 \\
\hline 1996 & 485900 & 488800 & -2900 \\
\hline 1997 & 499500 & 502300 & -2800 \\
\hline 1998 & 509400 & 517300 & -7900 \\
\hline 2000 & 530900 & 552400 & -21500 \\
\hline 2001 & 538000 & 567500 & -29500 \\
\hline 2002 & 546000 & 581200 & -35200 \\
\hline
\end{tabular}

Source : Statistical Abstract of Israel, 2003

13 La Galilée - où réside $46 \%$ des Palestiniens d'Israël - reste un espace de conquête démographique des plus difficiles à atteindre, notamment les sous-districts de St Jean d'Acre et de Jezréel. Pour le gouvernement israélien, cet affaiblissement de la croissance juive replace avec toute son acuité la question politique du développement d'une frontière interne en Israël même si des espaces d'immigration comme la vallée de Bet HaKerem - où est localisée Karmiel ${ }^{15}$ - ont contribué à repousser cette frontière 
en limitant l'expansion des villages arabes comme celui de Majd al-Krum (Yiftachel, 1998).

Présentant une force d'attraction équivalente à celle du district nord en regroupant environ $12 \%$ de l'immigration en 1990, le district sud, aujourd'hui bien moins stratégique qu'au sortir du premier conflit israélo-arabe, est l'espace qui aura le plus bénéficié du dynamisme migratoire. Au fil de la décennie quatre-vingt dix, il a vu sa capacité d'attraction croître jusqu'à en faire le premier espace de résidence des nouveaux immigrants. Avec respectivement 70300 et 58200 immigrants, Ashdod et Be'ersheva sont aujourd'hui les première et troisième cités d'accueil des nouveaux immigrants (figure 3).

Figure 3 : Principales villes de résidence des nouveaux immigrants (1989-2002) [afficher]

Cette dynamique s'explique par l'action conjuguée de la politique de constructions et des réseaux de solidarité au sein du groupe migrant, qui ont généré un ensemble de migrations internes favorable à ce district (Lipshitz, 1998 ; Berthomière, 2002). Lors du recensement général de population de 1995, les mobilités internes observées chez les immigrants ex-Soviétiques ont très nettement confirmé ce phénomène. Les exSoviétiques présentaient un taux de migrations internes pour le district sud de $47 \%$ soit près de six fois plus élevé que celui de la population totale d'Israël.

Acquis principalement aux dépens des districts de Tel-Aviv et de Jérusalem, ces mesures révèlent aussi que l'espace qui profite le plus de ces migrations internes est celui des colonies de Cisjordanie et de Gaza: 88 \%, soit une proportion deux fois supérieure à celle de la population juive globale ${ }^{16}$. Au cours de la décennie quatre-vingt dix Ariel, l'une des plus importantes implantations israéliennes de Cisjordanie, a vu s'installer environ 6000 nouveaux immigrants dont près des deux tiers par migration interne, principalement depuis Tel-Aviv (756 personnes) et ses villes satellites Petah Tiqwa (781), Rishon Leziyyon (146) mais aussi depuis Haïfa (210) et Jérusalem (170) (Berthomière, 2003a). Cinq années seulement après le lancement du programme de constructions pour l'accueil des nouveaux immigrants, le choix d'associer une vision toute stratégique de l'espace à ce programme apparaît alors comme une " réussite ».

De l'appropriation du territoire à l'« urbicide » : une nouvelle étape dans la géostratégie israélienne?

17 À observer sur l'ensemble de la décennie quatre-vingt dix cette dynamique migratoire au profit des implantations de Cisjordanie ${ }^{17}$, il convient de tempérer l'analyse. Un peu plus de 16000 ex-Soviétiques résident dans les colonies israéliennes (hors JérusalemEst) soit environ $2 \%$ du groupe migrant et moins de $8 \%$ de l'ensemble des colons (tableau 3).

Tableau 3 : Population des localités juives de Cisjordanie et de la bande de Gaza (1993-2001)

\begin{tabular}{|l|r|r|r|r|r|r|r|r|r|r|}
\hline & \multicolumn{1}{|c|}{1993} & \multicolumn{1}{|c|}{1994} & \multicolumn{1}{c|}{1995} & \multicolumn{1}{|c|}{1996} & \multicolumn{1}{c|}{1997} & \multicolumn{1}{c|}{1998} & \multicolumn{1}{c}{1999} & \multicolumn{1}{c}{2000} & \multicolumn{1}{c}{2001} & \multicolumn{1}{c|}{2002} \\
\hline Cisjordanie & 110900 & 121800 & 132000 & 142700 & 152000 & 163300 & 174000 & 187600 & 196700 & 212800 \\
\hline Gaza & 4800 & 5100 & 5300 & 5600 & 5700 & 6100 & 6300 & 6700 & 6900 & 7300 \\
\hline Total & 115700 & 126900 & 137300 & 148300 & 157700 & 169400 & 180300 & 194300 & 203600 & 220100 \\
\hline
\end{tabular}

Source : Statistical Abstract of Israel, 2003 
Sur les neuf colonies urbaines qui regroupent approximativement la moitié des colons israéliens, cinq constituent les principaux lieux d'établissement des ex-Soviétiques (tableau 4).

Tableau 4 : Colonies de Cisjordanie : population et immigration (1989-2002)

\begin{tabular}{|l|c|c|c|}
\hline $\begin{array}{l}\text { Colonies de } \\
\text { Cisjordanie }\end{array}$ & $\begin{array}{c}\text { Immigration } \\
\text { totale }\end{array}$ & $\begin{array}{c}\text { Population } \\
\text { totale }\end{array}$ & $\begin{array}{c}\text { Part des ex- } \\
\text { Soviétiques dans } \\
\text { 1'immigration totale }\end{array}$ \\
\hline Ariel & 7237 & 17263 & 96,6 \\
\hline Ma'ale Adoumim & 4055 & 27621 & 84,9 \\
\hline Modi'in Illith & 1664 & 21563 & 26,2 \\
\hline Betar Illith & 1463 & 19920 & 48,5 \\
\hline Kyriat Arba & 1401 & 6029 & 74,5 \\
\hline Karne shomeron & 942 & 6399 & 9,9 \\
\hline Efrat & 920 & 6969 & 30,2 \\
\hline Givat Zeev & 657 & 10827 & 27,9 \\
\hline Immanuel & 118 & 2837 & 32,2 \\
\hline
\end{tabular}

Source : Ministère de l'immigration et d'inté gration, 2003

19 En choisissant de s'installer dans ces «colonies-dortoirs " à proximité des bassins d'emplois de Tel-Aviv (pour Karne Shomeron et Ariel) et de Jérusalem (pour Givat Zeev et Ma'ale Adoumim), et plus globalement dans les implantations sises le long de la Ligne verte, il ressort que ces décisions d'installation relèvent bien plus d'une logique socioéconomique qu'idéologique. Soumis à de fortes difficultés d'insertion socioprofessionnelle, certains nouveaux immigrants n'ont eu comme choix que de s'installer dans ces implantations rendues économiquement accessibles par les subventions d'Etat.

Soulignons néanmoins que le renforcement démographique des colonies sises sur la Ligne verte a constitué l'un des principaux objectifs du Plan Seven Stars, mis sur pied par Ariel Sharon en 1991, alors qu'il était ministre de la Construction et du Logement. Ce plan avait d'ailleurs été établi "sous couvert d'absorption de la nouvelle vague d'immigration" (Legrain, 1996; Chagnollaud, 2000). De plus, à l'échelle des implantations, l'immigration aura contribué à asseoir la colonie d'Ariel dans sa fonction de sectionnement de la Samarie (Dieckhoff, 1987). Ariel présente aujourd'hui une population d'environ 17000 personnes, composée à $42 \%$ d'immigrants ${ }^{18}$. L'extension d'Ariel rend aujourd'hui bien difficile l'accès à la ville palestinienne de Salfit, pôle d'activités pour les villages d'alentour comme Kifl Haris ou Qira (Lein, 2002). Indéniablement, l'immigration récente et la politique de constructions dans les Territoires occupés ont impulsé une dynamique de croissance des colonies. Face à la crise du logement générée par la demande massive des immigrants des années 90 , de nombreux jeunes couples israéliens ont été entraînés dans le mouvement de migration interne en direction des implantations de Cisjordanie. L'attraction de ces colonies a été d'autant plus forte que le réseau routier les irriguant s'est nettement densifié. Globalement, les indicateurs en termes de constructions et de mobilités résidentielles tendent à confirmer que la décennie quatre-vingt dix aura donc été décisive dans la consolidation des implantations. En premier lieu, sur la période allant des «accords d'Oslo » à la seconde Intifada, le nombre d'unités de logements dans les colonies est passé de 20400 à 31400 soit une croissance de $54 \%$ (Lein, 2002) ${ }^{19}$. L'accroissement du parc immobilier a mené à une augmentation de $75 \%$ de la population des colonies ${ }^{20}$ et a 
permis, même si leur pouvoir d'attraction semble s'amoindrir, d'installer un régime démographique où la seule croissance naturelle assoit désormais leur ancrage territorial. Avec une croissance naturelle de 7100 personnes en 2002, les colonies de Cisjordanie et de Gaza affichaient un dynamisme démographique supérieur à celui des districts Nord et de Haïfa. En termes de croissance globale (croissance naturelle et balance migratoire cumulées), les Territoires occupés se classent en quatrième place juste derrière le district de Jérusalem (tableau 5).

Tableau 5 : Population juive et arabe : croissance et migration interne (2002)

\begin{tabular}{|c|c|c|c|c|c|c|c|c|}
\hline \multirow[b]{3}{*}{$\begin{array}{c}\text { Districts } \\
\text { et ternitoires } \\
\text { cocupés }\end{array}$} & \multirow[b]{3}{*}{$\begin{array}{c}\text { Croissance } \\
\text { annuelk } \\
(\%)\end{array}$} & \multirow[b]{3}{*}{$\begin{array}{c}\text { Population en } \\
\text { fin de période } \\
\text { (milliers) }\end{array}$} & \multirow[b]{3}{*}{$\begin{array}{c}\text { Croissance } \\
\text { totale } \\
\text { (milliers) }\end{array}$} & \multirow{2}{*}{\multicolumn{3}{|c|}{\begin{tabular}{|c|} 
Balance migratoire \\
dont \\
\end{tabular}}} & \multirow[b]{3}{*}{$\begin{array}{c}\text { Croissance } \\
\text { naturelle } \\
\text { (milliers) }\end{array}$} & \multirow[b]{3}{*}{$\begin{array}{l}\text { Population en } \\
\text { debut de période } \\
\text { (milliers) }\end{array}$} \\
\hline & & & & & & & & \\
\hline & & & & $\begin{array}{c}\text { Migrations } \\
\text { internes } \\
\text { (milliers) }\end{array}$ & \begin{tabular}{|c|} 
Immigrants \\
(milliers)
\end{tabular} & $\begin{array}{c}\text { Total } \\
\text { (milliers) }\end{array}$ & & \\
\hline & \multicolumn{8}{|c|}{ Population juive ${ }^{(1)}$} \\
\hline TOTAL & 1,6 & 5367,2 & 85,9 & - & 36,5 & 22,3 & 63,9 & 5281,3 \\
\hline Jérusalem & 1.8 & 564,4 & 10,0 & $-4,3$ & 4,3 & -15 & 115 & 554,4 \\
\hline Nond ${ }^{(4)}$ & 15 & 546,0 & 8,0 & $-2,6$ & 6,1 & 2,1 & 6,0 & 538,0 \\
\hline Hailfa & 0,6 & 653,2 & 3,7 & $-2,2$ & 4,9 & 0,7 & 3,0 & 649,5 \\
\hline Centre & 2,6 & 1418,4 & 35,3 & 15,1 & 6,8 & 18,4 & 169 & 1383,1 \\
\hline Tel Aviv & 0 & 1145,9 & 0 & $-10,9$ & 4,9 & $-9,4$ & 9,4 & 1146,0 \\
\hline Sud & 2,1 & 819,1 & 17,1 & 0,9 & 8,4 & 7,1 & 9,9 & 802,1 \\
\hline \multirow[t]{2}{*}{$\begin{array}{l}\text { Territoires } \\
\text { cocupes }^{(9)}\end{array}$} & 5,7 & 220,1 & 11,9 & 4,0 & 1,1 & 4,8 & 7,1 & 208,2 \\
\hline & \multicolumn{8}{|c|}{ Population arabe (2) } \\
\hline TOTAL & 3,0 & 1263,9 & 36,4 & - & 0,1 & $-0,8$ & 369 & 1227,5 \\
\hline Jénusalem & 3,0 & 229,7 & 6,8 & $-0,1$ & 0 & 0 & 67 & 222,9 \\
\hline Nord & 2,4 & 581,2 & 13,7 & $-0,2$ & 0,1 & $-1,2$ & 148 & 567,5 \\
\hline Hailfa & 2,7 & 185,7 & 4,9 & 0,1 & 0 & 0,1 & 4,8 & 180,7 \\
\hline Centre & 3,2 & 122,7 & 3,8 & 0 & 0 & 0 & 3,7 & 119,0 \\
\hline Tel Aviv & 2,6 & 15,2 & 0,4 & $-0,1$ & 0 & 0 & 0.3 & 14.8 \\
\hline Sud & 5,6 & 129,4 & 6,9 & 0,2 & 0 & 0.3 & 66 & 122,5 \\
\hline
\end{tabular}

(1) Donnée incluant la population chrétienne non-arabe et les personnes non classées par religion.

(2) Donnée incluant une partie des 4000 personnes en provenance du sud Liban (majoritairement des soldats de l'ex-ALS).

(3) Donnée non fournie pour la population arabe.

(4) Donnée incluant le Golan.

Source : Statistical Abstract of Israel, 2003

21 Haut lieu de cristallisation du conflit, Jérusalem a fait également l'objet de planification territoriale non sans répercussions sur le partage et le statut futur de la ville. Soumise depuis 1967 à de multiples changements, les frontières de Jérusalem délimitent un territoire au sein duquel les gouvernants israéliens cherchent à limiter l'érosion de la population juive (Legrain, 1996 ; Encel, 1998 ; Kaminker, 1998) ${ }^{21}$. Malgré l'imposition de quotas sur la construction de logements dans les quartiers palestiniens de la ville ${ }^{22}$ et le développement (par appropriation des terres) de nouveaux quartiers juifs dans la partie orientale, Jérusalem ne cesse de voir la part de sa population juive s'éroder (Kaminker, 1998 ; Lein, 2002) 23. Depuis le début des années quatre-vingt dix, la balance migratoire de la ville est de plus en plus déficitaire: en 1980, le solde migratoire affichait un taux de $-1,7 \%$ o habitants, en $1999,-12,5 \%$, en $2000,-12,6 \%$ et en $2001,-8,9 \%^{24}$. Inévitablement, le renouveau migratoire a été perçu comme le moyen de contrebalancer le contexte démographique déficitaire dans lequel se tient Jérusalem depuis le début des années quatre-vingt (DellaPergola, 1999).

Malgré plus de 57600 nouveaux immigrants (dont $57,5 \%$ originaires d'ex-URSS) entre 1989 et 2002, il convient de souligner que l'immigration n'a pas été le support d'un net sursaut de croissance et confirme donc le caractère pérenne de l'incapacité de 
Jérusalem à retenir sa population (Rivière-Tencer et al., 1997 ; DellaPergola, 1999). Pour exemple, sur la période précédente (1975-1988), alors que le flux migratoire était quatre fois moins élevé, la croissance brute de population juive s'établissait à un niveau quasi identique (environ 95000 personnes). Toutefois, à l'échelle des quartiers et de la géostratégie israélienne, cet afflux de migrants a soutenu la croissance juive dans la partie orientale de la ville. Du fait d'une très large offre en logements à des prix bien inférieurs à ceux pratiqués à Jérusalem-Ouest, les nouveaux quartiers juifs de la partie orientale de la ville ont accueilli une large part des nouveaux arrivants. Fin 1999, environ 16000 ex-Soviétiques résidaient dans ces quartiers, soit la moitié des immigrants « russes» de la ville. Au sud de la partie orientale de la ville, les quartiers de Gilo et de Talpiyot-est ont été les principaux lieux d'installation des immigrants tandis qu'au Nord, les quartiers de Ramot Alon (nord) mais surtout ceux de Pisgat Zeev et Neve Ya'acov ont rassemblé près de 9500 ex-Soviétiques (Berthomière, 2002). La croissance de ces quartiers - dopée par la forte immigration des années 1990 et 1991 a permis au gouvernement israélien de «proclamer » que la ville « réunifiée » était à majorité juive même dans sa partie Est.

Outre le non négligeable soutien démographique des immigrants, Israël semble atteindre une limite dans ses efforts pour contenir la croissance naturelle palestinienne. Alors que l'immigration était à ses meilleurs niveaux, la période 1987-1997 voit le taux de croissance démographique des Palestiniens reprendre le pas sur celui de la population juive après avoir connu un ralentissement sur la décennie précédente (tableau 6). En 2001, le bilan démographique depuis 1967 confirmait l'existence de cette limite avec une nette différence de croissance entre les deux protagonistes : $214 \%$ pour les Palestiniens, 129,9\% pour les Israéliens.

Tableau 6 : Jérusalem : population et croissance (1967-2001)

\begin{tabular}{|c|c|c|c|}
\hline Année & Population juive & $\begin{array}{c}\text { Population palestinienne } \\
\text { et autres }{ }^{(1)}\end{array}$ & Population totale \\
\hline 1967 & 197700 & 68600 & 266300 \\
\hline 1977 & 272300 & 103700 & 376000 \\
\hline Croissance sur 1967-1977 (\%) & 37,7 & 51,2 & 41,2 \\
\hline 1977 & 272300 & 103700 & 376000 \\
\hline 1987 & 346100 & 136500 & 482600 \\
\hline Croissance sur 1977-1987 (\%) & 27,1 & 31,6 & 28,4 \\
\hline 1987 & 346100 & 136500 & 482600 \\
\hline 1997 & 429100 & 193000 & 622100 \\
\hline Croissance sur 1987-1997 (\%) & 24 & 41,4 & 28,9 \\
\hline 2000 & 448800 & 208700 & 657500 \\
\hline 2001 & 454600 & 215400 & 670000 \\
\hline Croissance sur 1967-2001 (\%) & 129,9 & 214 & 151,6 \\
\hline
\end{tabular}

(1) Depuis 1996, la population * religion non classifiée $*$ est incluse dans la population totale et non dans la population * Arabe et autres * comme auparavant. Depuis 1998, la population juive intègre : les catégories * juifs $"$, * religions non classifiées $»$ et $*$ non Arabes chrétiens $»$. La catégorie « Arabe et autres w intè gre les Musulmans, Druzes et Arabes Chrétiens.

Source : Jerusalem Institute for Israel Studies

Le projet d'établissement d'une majorité juive à Jérusalem-Est apparaît donc bien difficile à atteindre. Pour 2001, selon différentes estimations, les populations juive et palestinienne de Jérusalem-Est s'élèveraient respectivement à 177000 et 215000 personnes. Par leur vigueur démographique, les Palestiniens confirment ainsi leur 
poids grandissant dans la population de la ville : $32,2 \%$ en 2001 soit $+6,4$ points de croissance depuis 1967.

Occultés par le «poids» du nombre, les autres «outils» de l'appropriation et du morcellement des terres palestiniennes apparaissent plus nettement aujourd'hui avec le moindre rôle de l'immigration et permettent de mieux saisir la difficulté pour les leaders palestiniens, mais aussi israéliens, d'aboutir à un accord viable. Que cela soit à l'échelle de Jérusalem ou des territoires de Cisjordanie et de Gaza, la géostratégie israélienne s'est accompagnée de mesures bien moins perceptibles qu'une nouvelle colonie mais toutes aussi négatives pour le processus de négociations. À Jérusalem, la confiscation des cartes de résidents constitue une pratique qui s'est multipliée au cours des dernières années ${ }^{25}$. Avec pour but de réduire la part de la population arabe de la ville, plus de 6300 cartes ont été saisies depuis 1967 dont près de la moitié après 1993 (figure 3). Suite à de multiples protestations sur le plan international, cette pratique a été freinée et 800 Palestiniens auraient recouvré leur droit en 2000 contre 183 en $1999^{26}$.

Figure 4 : Nombre de confiscation de cartes de résidents palestiniens de Jérusalem (1967-2000) [afficher]

À l'échelle des Territoires occupés, la destruction d'habitation ${ }^{27}$ et la saisie de terres oeuvrent de concert pour pousser au départ la population ainsi que pour préparer ou consolider l'espace des colonies (Debié et al., 2001 ; Lein, 2002 ; Stein, 2002) ${ }^{28}$. Une large part de ces terres a contribué au développement des routes de contournement (by-pass roads), qui enserrent aujourd'hui les territoires palestiniens de Cisjordanie et annihilent la continuité territoriale des territoires palestiniens. Selon Sylvie Fouet, cette « jungle d'asphalte » est composée d'une trentaine de routes, qui devraient aboutir (par la saisie de milliers de dunums ${ }^{29}$ à un réseau de $450 \mathrm{~km}$ de routes (Debié et al., 2001). A cet ensemble de pratiques visant à conquérir le territoire s'ajoute aujourd'hui la décision unilatérale de construire un mur de séparation isolant plus encore la population palestinienne. Long de plusieurs centaines de kilomètres, il redessine les limites de Jérusalem-Est en excluant par le tracé choisi une part non négligeable des résidents palestiniens de la ville comme ceux des quartiers de Abu Mrera ou Al-Azariya ${ }^{30}$. Les Palestiniens devront désormais chercher d'autres lieux pour scolariser leurs enfants et bien d'autres encore pour assurer leur vie quotidienne (des commerces aux hôpitaux).

Conquérir l'espace reste donc l'objectif premier de la géostratégie d'Israël. Conscient des limites de cette politique, le gouvernement d'Ariel Sharon a doublé sa géostratégie d'émiettement du territoire par des actions portant atteinte aux centralités palestiniennes, à "leur modernité $\|^{31}$. Entamée lors de la réoccupation des villes palestiniennes afin de « détruire l'infrastructure terroriste » et symbolisée par la bataille de Jénine en avril 2002, cette nouvelle étape de la stratégie israélienne est aujourd'hui perçue comme un urbicide ${ }^{32}$. Ces différents éléments de contrôle territorial ont profondément modifié les représentations géographiques du conflit chez les Israéliens. Les by-pass roads ont notamment contribué à créer l'illusion que la Ligne verte avait disparu et qu'il n'y a pas de population palestinienne dans les espaces que les routes sectionnent, risque que formulait Peace Now au début des années quatre-vingt dix ${ }^{33}$. Audelà, les récentes évolutions d'Israël dessinent en effet une société israélienne qui se pense de plus en plus comme non arabe, comme le suggère Alain Dieckhoff. Israël se trouve néanmoins dans une situation des plus délicates car il est certes possible de se 
penser non arabe mais bien difficile d'ignorer l'horizon démographique lorsque aucun des gouvernements successifs n'a su trouver les termes d'un accord depuis 1993.

L'avenir ne passe-t-il que par le nombre?

28 La logique du fait accompli laisse à penser qu'effectivement le nombre est au cœur de la géostratégie israélienne et, aujourd'hui, c'est une réalité concrète: pour exemple, la centaine d'implantations et « postes avancés » de Cisjordanie occupent « $1,7 \%$ des terres de Cisjordanie, leurs frontières municipales $6,8 \%$; les conseils régionaux en intègrent $35,5 \%$ soit au total $41,9 \%$ du territoire " (Lein, 2002). Mais le nombre joue aussi en défaveur des gouvernants israéliens: la courbe de l'immigration ne cesse de chuter (43 580 personnes en 2001, 33567 en 2002) alors que celle de l'émigration (yerida) serait en hausse $^{34}$ et, donnée encore plus "insupportable", les colonies connaîtraient un mouvement d'émigration vers les frontières de $1948^{35}$. De plus, la presse israélienne ouvre fréquemment ses colonnes à des scientifiques qui soulignent les horizons démographiques d'Israël. Les titres de ces articles sont évocateurs: How the Jewish majority can turn into a minority ou Doomsday demographer gets a hearing at the Prime Minister Office. Ariel Sharon n'ignore donc pas cette réalité du nombre mais témoigne de la permanence de sa stratégie lorsqu'il appelle à l'immigration d'un nouveau million d'immigrants. Faute d'immigrants, et les chances de voir une nouvelle vague migratoire sont faibles, il est certain que dans les configurations territoriales actuelles, la population juive deviendra démographiquement minoritaire dans la décennie à venir. L'ensemble des démographes israéliens atteste de cette perspective. Selon l'échelle des projections, Sergio DellaPergola estime que la population juive restera majoritaire (entre Méditerranée et Jourdain) jusqu'en 2010 (selon une hypothèse moyenne, cf. tableau 7) ou jusqu'en 2005 (selon une hypothèse haute). Nombreux sont aujourd'hui les démographes israéliens qui soulignent cet horizon démographique et qui appellent à la "séparation d'avec les Palestiniens » afin d'assurer une majorité juive sur le territoire d'Israël. Toujours selon ces projections, cette majorité dans un Etat d'Israël sans les territoires palestiniens tendra progressivement à se réduire pour atteindre une proportion de population juive entre 65,8 et $73 \%$ (selon les hypothèses) et à créer, selon Sergio DellaPergola (2000), une « situation d'État binational ».

Tableau 7 : Part des Juifs dans la population totale selon l'espace considéré en 2000, 2010, 2020 et 2050 (hypothèse moyenne en \%)

\begin{tabular}{|c|c|c|c|c|}
\hline $\begin{array}{l}\text { Projection } \\
\text { hypothise } \\
\text { moyenne }\end{array}$ & $\begin{array}{c}\text { Population juivel } \\
\text { Population totale } \\
\text { d'Tsraśl }\end{array}$ & $\begin{array}{c}\text { Population juive } \\
\text { élargie (1yPopulation totale } \\
\text { d'Isrä̌l }\end{array}$ & $\begin{array}{l}\text { Population juive/Population } \\
\text { d'Israel + Population des } \\
\text { Territoires palestiniers }\end{array}$ & $\begin{array}{c}\text { Population juive élargiel } \\
\text { Population d'Israël + Population } \\
\text { des Territoires palestiniens }\end{array}$ \\
\hline 2000 & 78,3 & 81,4 & 53,3 & 55,5 \\
\hline 2010 & 75,5 & 79,4 & 48,6 & 51,1 \\
\hline 2020 & 73,4 & 77,2 & 44,4 & 46,7 \\
\hline 2050 & 69,2 & 73,8 & 35,1 & 37,4 \\
\hline
\end{tabular}

(1) Cette catégorie est essentiellement composée des conjoints non-juifs des immigrants juifs (principalement originaires d'ex-URSS).

Source : Sergio DellaPergola, * Projections démographiques: combat de chiffres », Dossiers et recherches, $\mathrm{n}^{\circ} 90$, décembre 2000 , Paris : INED, p. 30.

Autant d'éléments qui soulignent que le véritable défi d'Israël réside dans sa capacité à se penser au-delà du nombre. La société israélienne est aujourd'hui décrite comme «schizophrénique» (Ram, 2000), incapable de trouver l'équilibre qui permettrait d'envisager son futur autrement qu'en termes «d'ingénierie » démographique ${ }^{36}$. Elle 
semble néanmoins être entrée dans une temporalité nouvelle, celle de la scission (cf. figure 2). La politique du territoire pratiquée depuis ces dernières années ne peut maintenant qu'évoluer vers une séparation. Mutuelle, unilatérale, inéquitable? La communauté internationale y a son rôle à jouer, la «feuille de route " est, était ? peutêtre, le «premier» pas. En 1999, Uri Ben-Eliezer semblait bien pessimiste quand il soulignait: "L'existence en Israël d'une société militaire puissante et efficace, dont la force repose sur des mécanismes de brouillage des limites entre armée et société et sur la pérennité du lien entre l'Armée et la politique, ne permet pas d'espérer l'émergence, dans un proche avenir, d'une société civile idéologiquement solide et véritablement soucieuse de paix " (Ben-Eliezer, 1999). Trois années se sont écoulées et l'avenir proche semble encore loin ; à tel point que la situation a poussé Avraham Burg, ancien Président de la Knesset (1999-2003) et ancien Président de l'Agence juive, à déclarer que «la révolution sioniste est morte $»^{37}$.

\section{BIBLIOGRAPHIE}

ALTERMAN Rachelle (2002) Planning in the face of crisis. Land use, housing and mass immigration in Israel, London, Routledge, 212 p. BEN-ELIEZER Uri (1999) « L'armée, la société et la nation-enarmes ", Cahiers de l'Orient, n 54, pp. 161-185. BERTHOMIÈRE William (1999) « L'immigration des travailleurs étrangers : miroir des mutations identitaires d'Israël », Les Cahiers de l'Orient, $\mathrm{n}^{\circ} 54$, pp. 117-133.

BERTHOMIÈRE William (2000) «L'État d'Israël de 1948 à l'après guerre froide : essai de lecture des interactions entre reconfigurations géopolitiques et régimes démographiques » in Régimes démographiques et territoires : les frontières en question (actes du 6. colloque international de l'AIDELF), Paris, Presses universitaires de France, pp. 461-473.

BERTHOMIÈRE William (2002) «Logiques de migrants versus logiques d'État : quels impacts sur la stratégie territoriale d'Israël », Espace, populations, sociétés, vol. n 1-2, pp. 37-52.

BERTHOMIÈRE William (2003a) « Immigration from the former Soviet Union: Measure of the impacts on Jerusalem and on the settlements of the West Bank and Gaza », The Arab World Geographer, vol. 6 (2), pp. 249-264.

BERTHOMIÈRE William (2003b) « Nouvelle Intifada, mondialisation et immigration de travailleurs étrangers : une nouvelle réalité sociale pour Israël », Maghreb-Machrek, vol. 177, pp. 79-99.

BERTHOMIÈRE William et Kamel Doraï (2002), « Israël-Palestine : populations, migrations, État(s) » in Dominique Borne et Jacques Scheibling (ed.), La Méditerranée, Paris, Hachette Supérieur, pp. 195-215.

CHAGNOLLAUD Jean-Paul (2000), « Les implantations israéliennes et l'avenir des territoires palestiniens » in Jean-Paul Chagnollaud et al. (ed.), Palestiniens et Israéliens : le moment de vérité, Paris, L'Harmattan (Les cahiers de Confluences), pp. 101-132.

DEBIÉ Franck et Sylvie FOUET (2001), La paix en miettes : Israël / Palestine, 1993-2000, Paris, Presses Universitaires de France, $442 \mathrm{p}$. 
36 Voir sur ce thème la réflexion de Myron Weiner et Michael Teitelbaum, Political Demography. Demographic Engineering, New York : Berghahn Books, 2001. 37 Cf. Le Monde daté du 10 septembre 2003.

DELLAPERGOLA Sergio (1999) «A new look at Jerusalem's future population Demography, multiculturalism and urban planning », European Population Conference, The Hague (30 August-3 September), $32 \mathrm{p}$.

DELLAPERGOLA Sergio (2000) «Projections démographiques : combats de chiffres » in INED (ed.), L'arrière-plan démographique de l'explosion de violence en Israël-Palestine, Paris, INED (Dossiers et documents, 90), pp. 25-31.

DIECKHOFF Alain (1987) Les espaces d'Israël, Paris, FEDN, 211 p.

DIECKHOFF Alain (1993) L'invention d'une nation : Israël et la modernité politique, Paris : Gallimard (Essais), $352 \mathrm{p}$.

DIECKHOFF Alain (ed.) (2001) Israël : une identité nationale en crise, Problèmes politiques et sociaux, Paris, La Documentation française, $80 \mathrm{p}$.

DUMPER Michael (1992) « Israeli settlement in the Old City of Jerusalem », Journal of Palestine Studies, vol. XXI (4), pp. 32-53.

DUMPER Michael (1997) The Politics of Jerusalem, New York, Columbia University Press, 367 p.

DUMPER Michael (2000) «Colons et colonies dans la vieille ville de Jérusalem : 1980-2000 » in Farouk Mardam-Bey, Elias Sanbar (ed.), Jérusalem. Le sacré et le politique, Arles, Sindbad, pp. 273-302.

EISENSTADT S.N. (1985) The transformation of the Israeli society. An essay of interpretation, London, Weidenfeld and Nicolson, $590 \mathrm{p}$.

EISENSTADT S.N. (1986) The development of the ethnic problem in Israeli society, Jerusalem, The Jerusalem Institute for Israel Studies, $45 \mathrm{p}$.

ELLMAN M. et LAACHER Smaïn (2003) Migrant workers in Israel. A contemporary form of slavery, Paris, Fédération internationale des ligues des droits de l'Homme-FIDH et Réseau euroméditerranéen des droits de l'Homme (REMDH), 48 p.

ENCEL Frédéric (1998) Géopolitique de Jérusalem, Paris, Flammarion (Essais), 283 p.

FALAH Walid-Ghazi (1991) « Israeli judaization policy in Galilee », Journal of Palestine Studies, vol. XX (4), pp. 69-85.

FALAH Walid-Ghazi (2003) « Dynamics and patterns of the shrinking of Arab lands in Palestine », Political Geography, vol. 22, pp. 179-209.

GREILSAMMER Ilan (1998) La nouvelle histoire d'Israël. Essai sur une identité nationale, Paris, Gallimard (NRF essais), $584 \mathrm{p}$.

KAMINKER Sarah (1998) « Jérusalem-Est, un cas d'école en matière de planification politique » in R. Dhoquois-Cohen et al. (ed.), Jérusalem, ville ouverte, Paris, L'Harmattan (Les cahiers de Confluences), pp. 135-140.

KAPELIOUK Amnon (1975) Israël : la fin des mythes, Paris, Albin Michel, 318 p.

KIMMERLING Baruch (2001) The invention and decline of Israeliness. State, society, and the military, Berkeley, University of California Press, $268 \mathrm{p}$.

KLEIN Claude (1999) Israël, État en quête d'identité, Firenze, Casterman-Giunti (xx.Siècle), 125 p. 
LAURENS Henry (1999) Paix et Guerre au Moyen-Orient. L'Orient arabe et le monde de 1945 à nos jours, Paris, Armand Colin, 560 p.

LEGRAIN Jean-François (1996) « Judaïsation et démembrement : politiques israéliennes du territoire en Cisjordanie-Gaza (1967-1995) », Maghreb-Machrek, vol. 152, pp. 42-78.

LEGRAIN Jean-François (2000), « Retour sur les accords israélo-palestiniens (1993-2000) », Maghreb-Machrek, vol. 170, pp. 96-125.

LEIN Yehezkel (2002) Land grab. Israel's settlement policy in the West Bank (Draft version), Jerusalem: B'Tselem - The Israeli information center for Human rights in the Occupied territories, $103 \mathrm{p}$.

LIPSHITZ Gabriel (1998) Country on the move: Migration to and within Israel, 1948-1995, Dordrecht, Kluwer Academic Publishers (The GeoJournal Library), 167 p.

POIRIER Véronique (1998) Ashkénazes et Séfarades : une étude comparée de leurs relations en France et en Israël (années 1950-1990), Paris, Cerf, 312 p.

PORTNOV Boris (1998) « The effects of housing on migrations in Israel: 1988-1994 », Journal of Population Economics (Spring), pp. 379-394.

RAM Uri (1995) The changing agenda of the Israeli sociology: theory, ideology, and identity, Albany, State University of New York Press (SUNY series in Israeli studies), 232 p.

RAM Uri (2000) «The Promised land of business opportunities » : Liberal post-zionism in the glocal age » in G. Shafir and Y. Peled (ed.), The new Israel: Peacemaking and liberalization, Boulder, Westview Press, pp. 217-242.

RIVIERE-TENCER V. et ATTAL A. (1997) Jérusalem : destin d'une métropole, Paris, L'Harmattan (Comprendre le Moyen-Orient), $411 \mathrm{p}$.

SEGEV Tom (1998) Les premiers Israéliens, Paris, Calmann-Levy, 418 p.

SILBERSTEIN Laurence J. (ed.) (1999) The Postzionism debates. Knowledge and power in Israeli culture, London, Routledge, 275 p.

STEIN Yael (2002) Policy of destruction: House demolitions and destruction of agricultural land in the Gaza strip, Jerusalem: B’Tselem - The Israeli information center for Human rights in the Occupied territories, $42 \mathrm{p}$.

YAFFE Nurit et Dorith TAL (2002) The Arab population of Israel, Jerusalem, Central Bureau of Statistics (Statistilite $\left.\mathrm{n}^{\circ} 27\right), 12 \mathrm{p}$.

YIFTACHEL Oren (1992) Planning a mixed region in Israel, Aldershot, Avebury, $367 \mathrm{p}$.

YIFTACHEL Oren (1998) « The internal frontier: Territorial control and Ethnic relations in Israel » in O. Yiftachel and A. Meir (ed.), Ethnic frontiers and peripheries. Landscapes of development and inequality in Israel, Boulder, Westview Press, pp. 39-68.

\section{NOTES}

1. -Chiffres publiés début septembre 2003 par The Palestinian Monitor et le Magen David Adom. Selon les données de The Palestinian Monitor, 331 victimes palestiniennes seraient âgées de moins de quinze ans. A ces derniers s'ajoutent plus de 37000 blessés dont 2 500 garderont une infirmité.

2. -Voir l'article «Sharon wants $1 m$ new Jews for Israel » publié dans l'édition du 7 novembre $2001 \mathrm{du}$ Guardian. 
3. Cité par Tom Segev (1998).

4. Cf. Le Monde daté du 16 janvier 1990.

5. -Le Gouch Emounim ou « Bloc de la foi », groupe de sionistes-religieux créé au lendemain de la Guerre du Kippour (1973), prône au nom d'un « messianisme territorial » l'implantation dans les territoires afin « d'accélérer le processus de rédemption » (Dieckhoff, 1993).

6. -Cité par Uri Ben-Eliezer (1999).

7. -Concept emprunté à Arjun Appaduraï. Voir notamment "Global Ethnoscapes : Notes and Queries for a Transnational Anthropology" in Fox, R (ed.) (1991), Recapturing Anthropology: Working in the Present, Santa Fe, School of American Research Press. 8. -Voir le rapport The impact of closure and other mobility restrictions on Palestinian productive activities, New York: United Nations (Office of the United Nations special coordinator, October 2002).

9. -Selon Ha'aretz (édition du 20 avril 2003), de septembre 2002 - date de l'établissement de la police de l'immigration - à avril 2003, près de 15000 travailleurs étrangers auraient été expulsés (soit un peu plus de $10 \%$ de la population estimée de travailleurs étrangers irréguliers). En 2002, ces expulsés ont passé en moyenne 18 jours en prison avant leur expulsion et environ 200 d'entre eux plus de trois mois. Les données révélées par le Ministère de l'Emploi montrent également la diversité des origines des travailleurs étrangers puisque le groupe est composé de $23 \%$ de Roumains, $13 \%$ de Chinois, $9 \%$ de Turcs, $18 \%$ d'Indiens, Jordaniens et d'Ukrainiens ( $6 \%$ chacun) et $10 \%$ de Thaïlandais et Moldaves (5\% chacun) (Ha'aretz, édition du 15 avril 2003). 10. -Lapierre Nicole, « Le modèle de la diaspora juive en Europe ", in Gallissot R., ed., 1993, Pluralisme culturel en Europe. Culture(s) européenne(s) et culture(s) des diasporas, Paris : L'Harmattan (L'Homme et la Société), p. 32

11. -Déclaration de Golda Meir, alors Premier ministre d'Israël, lors d'une cérémonie organisée pour de nouveaux immigrants soviétiques sur le plateau du Golan (citation de l'article publié dans le journal Maariv, 26 septembre 1971).

12. -Cet espace septentrional a toujours vu les plans régionaux de développement de la présence juive en Galilée poursuivre trois axes stratégiques dans le but ultime de la maîtriser démographiquement : «1. l'encerclement des aires arabes densément peuplées par l'implantation de villages juifs (décennies 40 et 50 ) ; 2 . la pénétration limitée de ces aires de peuplement arabe par la création d'implantations urbaines principalement (décennie 60 et début des années 70 ) ; 3 . la pénétration extensive de ces espaces arabes par la multiplication des implantations rurales » (Yiftachel, 1992). 13. -Sur la période 1989-2002, les juifs ex-soviétiques ont composé 82,5\% des 1063023 immigrants enregistrés.

14. - Ce taux de croissance est parmi les plus élevés au monde. Selon (Yaffe et al., 2002), au plan régional, il est inférieur à celui de l'Autorité palestinienne (4,3\% par an) et supérieur à ceux de Syrie et Jordanie (2,4\% par an).

15. -Fin 2002, Karmiel comptait 47573 habitants dont 18197 immigrants arrivés depuis 1989, soit plus tiers de la population totale. Rappelons que les 1063023 immigrants enregistrés sur cette période composent 15,6 \% de la population d'Israël.

16. -Voir Immigrant population from Former Soviet Union 1995. Demographics trends, Jerusalem : Central Bureau of Statistics ( $n^{\circ}$ 1076), 1998.

17. -Les colonies de la Bande de Gaza n'ont été que très faiblement concernées par l'immigration massive d'ex-URSS.

18. -Données du Ministère de l'Immigration pour la période du 1.1.1989 au 31.12.2002. 
19. -Y. Lein (2002) rappelle dans son rapport que l'activité de constructions a atteint son pic d'intensité sous le gouvernement Barak avec 4800 nouvelles unités mises en chantier en 2000.

20. -La population des colonies affiche un taux de croissance annuel de $7 \%$ (2,5\% pour l'ensemble de la population juive d'Israël).

21. -Concernant la vieille ville de Jérusalem, voir les publications du Passia et les travaux de Michael Dumper (Dumper, 1992, 1997, 2000)

22. -Selon B'Tselem, de 1967 à fin 2000, sur les 97700 unités d'habitations construites, seulement $18 \%$ ont été attribuées à la population palestinienne.

23. -Voir aussi le rapport The Status of Jerusalem, New York: United Nations (Division for Palestine Rights), 31 August 1997.

24. -Soulignons que depuis 1982, le premier espace d'accueil des Hiérosolymitains sont les Territoires occupés ( 5807 personnes en 2000 contre 2356 vers le district de Tel Aviv et 2273 vers le district Centre).

25. -Voir notamment le rapport Forced eviction and dispossession of Palestinians in Occupied Jerusalem by current Israeli policies (1999-2000), Jerusalem: The Orient House (Department of International Relations).

26. -Ha'aretz, 2 avril 2001.

27. -Voir les informations fournies par le Comité israélien contre les démolitions (http://www.icahd.org)

28. -Pour analyse sur le temps long de la question de l'appropriation des terres palestiniennes, voir l'article de (Falah, 2003).

29. -Un dunum est égal à un dixième d'hectare.

30. -Cf. l'article "East-Jerusalem's Arabs find fence will literally cut their lives in half ", Ha'aretz, September 4, 2003 et “Actually, it's called Spartaheid”, Ha'aretz, September 16, 2003.

31. -Voir l'article de Stephen Graham «Clean territory» : urbicide in the West Bank disponible sur le site internet de The Israeli Committee Against House Demolitions. Selon ce comité, plus de 8000 maisons palestiniennes auraient été détruites depuis 1967 (Jérusalem-Est incluse)

32. -Voir l'article de Sari Hanafi (2003), «Jenin Camp: paradigm of spaso-cide », Al Ayyam Newspaper, Ramallah, $15^{\text {th }}$ April.

33. -Voir le rapport By-Pass Road Construction in the West Bank. The End of the Dream of Palestinian Sovereignty rédigé par The Palestinian Society for the Protection of Human Rights and the Environment (28 February 1996).

34. -Voir Le Monde daté du 28 septembre 2001.

35. -Voir l'article Settlers voted with their feet, Ha'aretz, March 6, 2003.

36. -Voir sur ce thème la réflexion de Myron Weiner et Michael Teitelbaum, Political Demography. Demographic Engineering, New York : Berghahn Books, 2001.

37. -Cf. Le Monde daté du 10 septembre 2003. 


\section{RÉSUMÉS}

Après avoir tant espéré dans la Déclaration de principes d'Oslo (1993), les années quatre-vingt dix se sont refermées sur une situation d'enlisement du processus de paix qu'est venue confirmer le second soulèvement palestinien - l'Intifada Al Aqsa-- et le recours à des actions militaires de plus en plus massives du coté israélien. Les échecs successifs des négociations (à Camp David, 2000, à Taba, 2001) ont eu pour conséquences de replacer le nombre au coeur du conflit israélopalestinien. C'est ce recentrage sur la donne démographique, qui avait perdu de sa centralité lors des années quatre-vingt dix, que cet article tente d'analyser pour mieux saisir la réalité de la stratégie territoriale d'Israël.

The Return of the number : permanences and limits of the Israeli territorial strategy. After having hoped so much in the Declaration of Principles (Oslo 1- 1993), the Nineties have been closed on the sentiment that the Peace process was in a deadlock. This statement was confirmed by the second Palestinian uprising -the Intifada Al Aqsa- and, on the Israeli side, by resorting to massive military actions. The successive failures of the negotiations (Camp David, 2000, Taba, 2001) resulted in to replace the number in a key position in the Israeli-Palestinian conflict. This is this re-emergence of the demographic question, which had lost its key position during the Nineties that this article proposes to analyse for better seizing the reality of the territorial strategy of Israel.

El retorno del número: permanencias y límites de la estrategia territorial israelí. Tras las grandes expectativas que la Declaración de Oslo (1993) generó, los años noventa han sido el escenario de un repliegue hacia una situación de punto muerto en el proceso de paz. Este repliegue se ha visto confirmado por el segundo sublevamiento palestino - la Intifada Al Aqsa - y por el recurso a acciones militares masivas por parte de los israelíes. Como consecuencia de los sucesivos fracasos de las negociaciones (Camp David en 2000, Taba en 2001), el número se ha convertido en el centro del conflicto israelí-palestino. El artículo analiza este giro hacia la cuestión demográfica (rol clave que la demografía había perdido durante los noventa), con el fin de comprender la estrategia territorial de Israel.

\section{INDEX}

Mots-clés : aliya, croissance démographique, histoire, identité nationale, immigration, prospective, répartition géographique, retour, territoire, Juifs

Index géographique : Israël

\section{AUTEUR}

\section{WILLIAM BERTHOMIÈRE}

Chargé de recherche CNRS, équipe MIGRINTER (Unité mixte de recherche 6588 CNRS, Maison des Sciences de l'Homme et de la Société, 99 av du Recteur Pineau, Poitiers, France. william.berthomiere@univ-poitiers.fr 